\title{
Protein interactions in situ
}

A twist on the MAPPIT method enables studying protein-protein interactions within living mammalian cells.

Among the many ways to monitor interactions between proteins, methods that can be used within living mammalian cells, that apply to membrane proteins and that could be amenable to large-scale screening are the exception rather than the rule. In reporting a recent modification of their mammalian protein-protein interaction trap, or MAPPIT, method, Jan Tavernier and colleagues at Ghent University and VIB, Belgium, describe an approach they term KISS (kinase-substrate sensor) that fulfills these criteria.

In MAPPIT, functional complementation of a cytokine receptor upon interaction of a 'bait' and 'prey' results in reporter activity via phosphorylated STAT3. The researchers realized that the TYK2 kinase domain has an inherent ability to phosphor- ylate a gp130 sequence fused to it in cis. By designing the assay such that the kinase (TYK2) and substrate (gp130) sequences are provided in trans, fused to bait and prey respectively, they made the luciferase reporter signal dependent on bait-prey interaction.

Because no component of KISS is targeted to the plasma membrane, it is not limited to monitoring soluble proteins. Indeed, Tavernier and colleagues demonstrated that the assay detects oligomerization of the ERN1 protein in the endoplasmic reticulum membrane upon triggering of the unfolded protein response. They also demonstrated that KISS detects interactions between cytosolic proteins, between $\mathrm{G}$ protein-coupled receptors and $\beta$-arrestin and, in a modification of the basic assay, between small molecules and their protein targets.

The researchers used previously established positive and random reference sets to benchmark the performance of KISS. As has been reported for other approaches, KISS detects about $30 \%$ of the positive reference set, with low false positives. Also, it detects a different set of interactors than other assays do. It should be noted, however, that the reference sets used for benchmarking are not enriched for membrane proteins.

As Tavernier and colleagues acknowledge, the assay has some limitations: it cannot be used for studies that directly affect STAT3 signaling, and it is limited in its ability to study dynamic processes. Nonetheless, having a complementary way to monitor protein interactions within living mammalian cells, particularly those of membrane proteins, is a welcome addition to the methodological repertoire.

Natalie de Souza

RESEARCH PAPERS

Lievens, S. et al. KISS, a mammalian in situ protein interaction sensor. Mol. Cell. Proteomics doi:10.1074/ mcp.M114.041087 (25 August 2014). 